\title{
CXCL4L1-fibstatin cooperation inhibits tumor angiogenesis, lymphangiogenesis and metastasis
}

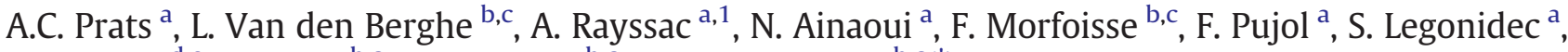 \\ A. Bikfalvi ${ }^{\mathrm{d}, \mathrm{e}}$, H. Prats ${ }^{\mathrm{b}, \mathrm{c}}$, S. Pyronnet ${ }^{\mathrm{b}, \mathrm{c}}$, B. Garmy-Susini ${ }^{\mathrm{b}, \mathrm{c}, *}$ \\ a Université de Toulouse, UPS, TRADGENE, EA4554, F-31432 Toulouse, France \\ b Inserm, U1037, F-31432 Toulouse, France \\ c Université de Toulouse, UPS, Cancer Research Center of Toulouse, F-31432 Toulouse, France \\ 'Inserm, U920, F-33405 Talence, France \\ e Université de Bordeaux 1, F-33405 Talence, France
}

\section{A R T I C L E I N F O}

\section{Article history:}

Accepted 20 May 2013

Available online 4 June 2013

\begin{abstract}
A B S T R A C T
Anti-angiogenic and anti-lymphangiogenic drugs slow tumor progression and dissemination. However, an important difficulty is that a tumor reacts and compensates to obtain the blood supply needed for tumor growth and lymphatic vessels to escape to distant loci. Therefore, there is a growing consensus on the requirement of multiple anti-(lymph)angiogenic molecules to stop cell invasion efficiently.

Here we studied the cooperation between endogenous anti-angiogenic molecules, endostatin and fibstatin, and a chemokine, the Platelet Factor-4 variant 1, CXCL4L1. Anti-angiogenic factors were co-expressed by IRES-based bicistronic vectors and their cooperation was analyzed either by local delivery following transduction of pancreatic adenocarcinoma cells with lentivectors, or by distant delivery resulting from intramuscular administration in vivo of adeno-associated virus derived vectors followed by tumor subcutaneous injection. In this study, fibstatin and CXCL4L1 cooperate to inhibit endothelial cell proliferation, migration and tubulogenesis in vitro. No synergistic effect was found for fibstatin-endostatin combination. Importantly, we demonstrated for the first time that fibstatin and CXCL4L1 not only inhibit in vivo angiogenesis, but also lymphangiogenesis and tumor spread to the lymph nodes, whereas no beneficial effect was found on tumor growth inhibition using molecule combinations compared to molecules alone. These data reveal the synergy of CXCL4L1 and fibstatin in inhibition of tumor angiogenesis, lymphangiogenesis and metastasis and highlight the potential of IRES-based vectors to develop anti-metastasis combined gene therapies.
\end{abstract}

(c) 2013 Elsevier Inc. All rights reserved.

\section{Introduction}

Angiogenesis promotes tumor growth, while both angiogenesis and lymphangiogenesis facilitate tumor dissemination by enhancing transport of tumor cells to new sites (Mumprecht and Detmar, 2009). Tumor spread is a leading cause of cancer-related mortality and morbidity, and both tumor cell intrinsic and extrinsic factors can promote tumor cells dissemination (Nagy et al., 2002; Skobe et al., 2001; Stacker et al., 2001). Metastases can be detected in draining lymph nodes and, for many tumors including pancreatic, breast cancer and melanoma, progress from lymph nodes to distant sites (GarmySusini et al., 2010; Renyi-Vamos et al., 2005). In the absence of neovasculature, tumors remain dormant (Nierodzik and Karpatkin, 2006). Thus, novel antiangiogenic treatment strategies that control

\footnotetext{
* Corresponding author at: INSERM U1037, 1, av. Jean Poulhes, BP 84225, 31432 Toulouse cedex 4, France. Fax: + 33561325622

E-mail address: barbara.garmy-susini@inserm.fr (B. Garmy-Susini).

${ }^{1}$ Present address: Cayla Invivogen, Toulouse, France.
}

tumor growth are under intense investigation. However, recent studies have shown evidences that treatment with anti-angiogenic molecules elicits malignant progression of tumors and accelerates metastasis suggesting that tumor cells escape the anti-angiogenic therapy (PaezRibes et al., 2009). As tumor progression is associated with expression of increasing numbers of pro-angiogenic and lymphangiogenic factors, the blockade of tumor invasion may require several anti-(lymph)angiogenic agents to simultaneously attack this multiple redundant process. In this study, we analyzed the combined effect of endogenous antiangiogenic molecules endostatin, fibstatin and CXCL4L1. Endostatin is a proteolytic polypeptide derived from collagen XVIII (O'Reilly et al., 1997), fibstatin, a proteolytic polypeptide derived from fibronectin (Bossard et al., 2004), and CXCL4L1 (PF4V1) a chemokine, the product of the human nonallelic variant gene of CXCL4 (PF4) (Dubrac et al., 2010). Those anti-angiogenic molecules were chosen for their properties to inhibit angiogenesis by targeting different signaling pathways. Endostatin interferes with vascular endothelial growth factor (VEGF) signaling. Fibstatin specifically interacts with the angiogenic (and lymphangiogenic) fibroblast growth factor 2 (FGF2) (Bossard et al., 
2004). CXCL4L1 is a small inducible cytokine binding to $G$ proteincoupled receptors (GPCR) recently described as an anti-angiogenic chemokine (Struyf et al., 2007).

To deliver combined molecules to tumor cells, we used gene transfer vectors based on internal ribosome entry sites (IRESs). IRESs represent useful tools to co-express combinations of two or more molecules in a controlled ratio (Rayssac et al., 2009). We compared the effect of local delivery (tumor cells transduced with lentivectors) or distant delivery (intramuscular injection of recombinant adeno-associated virus derived vectors, rAAV) to target both primary tumor and distant loci.

Our study shows a strong cooperative effect of fibstatin and CXCL4L1, but not of fibstatin and endostatin in inhibition of endothelial cell tubulogenesis, proliferation and migration in vitro as well as in the blockade of tumoral angiogenesis in vivo. Interestingly, we demonstrated for the first time the anti-lymphangiogenic effect of fibstatin and CXCL4L1. Then, this study revealed the importance of fibstatin and CXCL4L1 synergistic cooperation on the inhibition of tumor spread.

Indeed, we obtained stronger effect with lower amount of proteins using bicistronic vectors, the cooperative effect of fibstatin and CXCL4L1 was not observed on progression of the primary tumor, but was significant in inhibition of tumor invasion in lymph nodes.

\section{Materials and methods}

Mice study

Animal experiments were conducted in accordance with the recommendations of the European Convention for the Protection of Vertebrate Animals used for experimentation. All animal experiments were performed according to the INSERM IACUC guidelines for laboratory animals' husbandry and have been approved by the local branch Inserm Rangueil-Purpan of the Midi-Pyrénées ethics committee (protocol no. 088581114).

\section{Lentivector construction and transduction}

The cDNAs coding human endostatin, fibstatin and CXCL4L1 (PF4V1), were subcloned into the lentivector PTRIP-DU3-CMV-MCS derived from the pTRIP-DU3-EF1a-EGFP (Sirven et al., 2001). The VEGF secretion signal sequence and the HA tag were added to the N-terminal and C-terminal, respectively, of endostatin and fibstatin. The EF1a promoter was replaced by the cytomegalovirus (CMV) promoter and a multiple cloning site was inserted in place of the green fluorescent protein (GFP). This generated the monocistronic lentivectors pTRIP-fibstatin, -endostatin, and -CXCL4L1 respectively. To generate the bicistronic lentivectors, the cDNAs were subcloned by pairs and separated by the FGF1 IRES, resulting in the lentivectors pTRIP-fibstatin-endostatin, -fibstatin-CXCL4L1 and -CXCL4L1-fibstatin (Fig. 1). The six mono- or bicistronic lentivectors were produced using the tri-transfection procedure using the plasmids pLvPack and pLvVSVg (Sigma-Aldrich, SaintQuentin Fallavier, France), and were evaluated for their ability to transduce pancreatic adenocarcinoma cell line (Capan-1). In each case, GFP was used as a reporter transgene, and GFP FACS analysis was used to visualize transduced cells in vitro (Suppl. Fig. 1).

A total of $6 \times 10^{5}$ Capan- 1 cells were plated in 6 -well plates and transduced overnight in $1 \mathrm{~mL}$ of transduction medium (OptiMEM, Life Technologies SAS, Saint Aubin, France) containing $6 \mu \mathrm{g} / \mathrm{mL}$ protamine sulfate in the presence of purified lentiviral vector (MOI 0.8). Medium was changed and cells were collected $48 \mathrm{~h}$ after transduction. EGFPpositive cells were quantified by flow cytometry analysis on FACScalibur (Becton Dickinson SAS, Le Pont de Claix, France).

\section{rAAV construction and transduction}

The mono- and bicistronic cassettes coding the anti-angiogenic factors described above were subcloned into the vector pAAV-MCS
(Stratagene, Massy, France), resulting in three monocistronic recombinant adeno-associated viruses (rAAV): pAAV-endostatin, pAAVfibstatin, pAAV-CXCL4L1; and two bicistronic rAAVs: pAAV-fibstatinendostatin, pAAV-CXCL4L1-fibstatin (Fig. 1). Serotype 1/2 rAAVs were produced using the tri-transfection procedure using the plasmids pHelper (Stratagene) and pDP1rs (Plasmidfactory), and injected in the mice tibialis and gastrocnemius skeletal muscles. rAAV administration to each mouse $\left(100 \mu \mathrm{L}, 5.10^{11} \mathrm{pfu} / \mathrm{mL}\right)$ was divided into four injections: $20 \mu \mathrm{L}$ were injected in each tibialis and $30 \mu \mathrm{L}$ in each gastrocnemius. rAAVs were evaluated for their ability to transduce mouse skeletal muscle. As a control, we constructed and produced a bicistronic rAAV expressing, under the control of the CMV promoter, the renilla luciferase and the firefly luciferase (Luc + ) separated by the FGF1 IRES: pTRIPCRF1AL + . Firefly luciferase immunohistological analysis was used to visualize transduced cells in vivo (not shown).

\section{Reagents}

Recombinant human FGF-2 was from R\&D Systems (Minneapolis, MN). Rabbit anti-mouse Lyve-1 antibody (RDI-103PA50) was from Research Diagnostics Incorporated (Concord, MA). Rat anti-mouse CD31 (MEC 13.3) was from BD Bioscience (San Diego, CA). Goat antiPAN Cytokeratin, mouse anti-HA, donkey anti-rabbit and rat IgGs conjugated with DyLights Fluors 488, 568 were from TebuBio. Growth factor depleted Matrigel was from Becton-Dickinson. Anti-human CXCL4 monoclonal antibody (mAb7952) is from R\&D Systems (Minneapolis, $\mathrm{MN})$.

\section{Cell culture}

HUVECs (Promocell, Heidelberg, Germany) were cultured in endothelial growth medium (EGM-2) containing 2\% FBS (Promocell). Capan-1 pancreatic ductal carcinoma cells were obtained from the $\mathrm{NCI}$ ATCC and each was cultured in RPMI containing 10\% FBS and antibiotics.

\section{3-(4,5-Dimethylthiazol-2-yl)-2,5-diphenyltetrazolium bromide (MTT)} assays

HUVEC cells (2000 cells per well) were incubated in triplicate in a 96-well plate in the presence or absence of lentiviral-transduced cells supernatants containing $10 \mathrm{ng} / \mathrm{mL}$ FGF2 for $48 \mathrm{~h}$ (R\&D Systems, Minneapolis, MN). The ability of anti-angiogenic molecules to suppress cell growth was determined by MTT cell proliferation assays according to manufacturer instructions (Sigma-Aldrich). Experiments were performed three times in triplicate.

\section{Migration assays}

Confluent HUVECs monolayers were scratched using a $20 \mu \mathrm{L}$ pipette tip. Plates were washed with PBS and incubated with lentiviraltransduced cells supernatants containing $20 \mathrm{ng} / \mathrm{mL}$ FGF2 for $24 \mathrm{~h}$. Experiments were performed three times in triplicate.

\section{Endothelial cell tubulogenesis assay in matrigel}

HUVECs ( $5.10^{4}$ cells) were added to 4 wells chamber slides containing $300 \mu \mathrm{L}$ Matrigel in the presence of capan- 1 mono- and bicistronic transuduced cells conditioned media containing $10 \mathrm{ng} / \mathrm{ml} \mathrm{FGF2.} \mathrm{Cham-}$ ber slides were incubated at $37{ }^{\circ} \mathrm{C}$ for $24 \mathrm{~h}$. The mean number of vessel branch points $+/-$ SEM was determined for triplicate samples. Experiments were performed three times.

\section{Immunohistochemistry}

Tumors, draining (inguinal) lymph nodes and muscles were embedded in OCT compound (Tissue-Tek; Sakura Finetek, Torrance, 
A

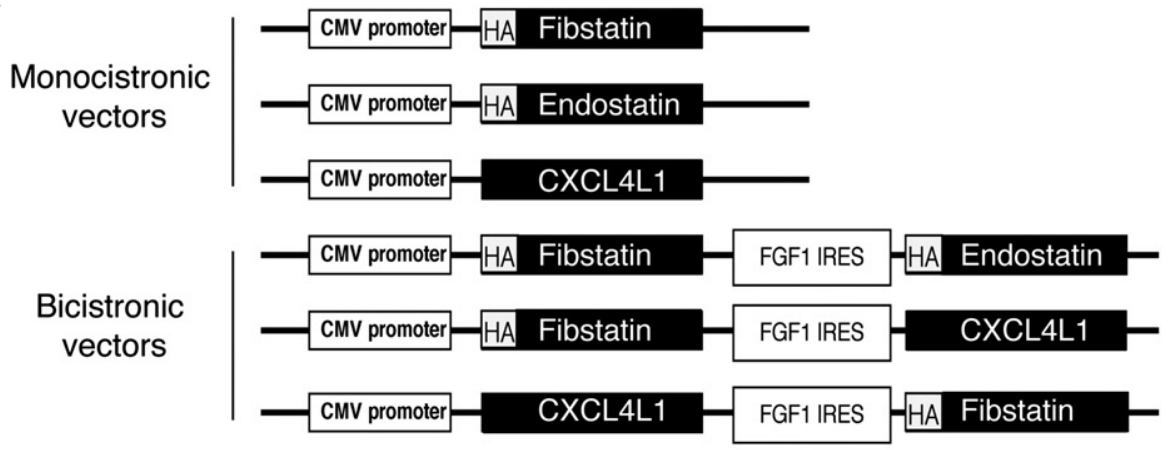

B

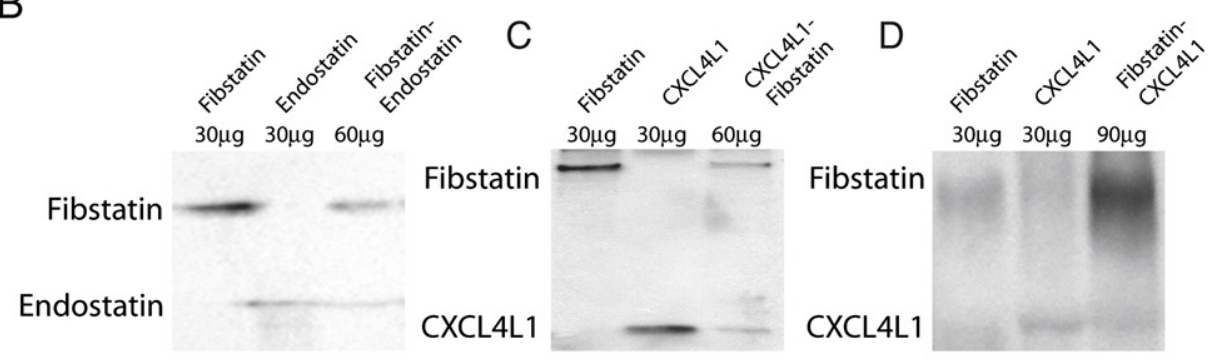

E
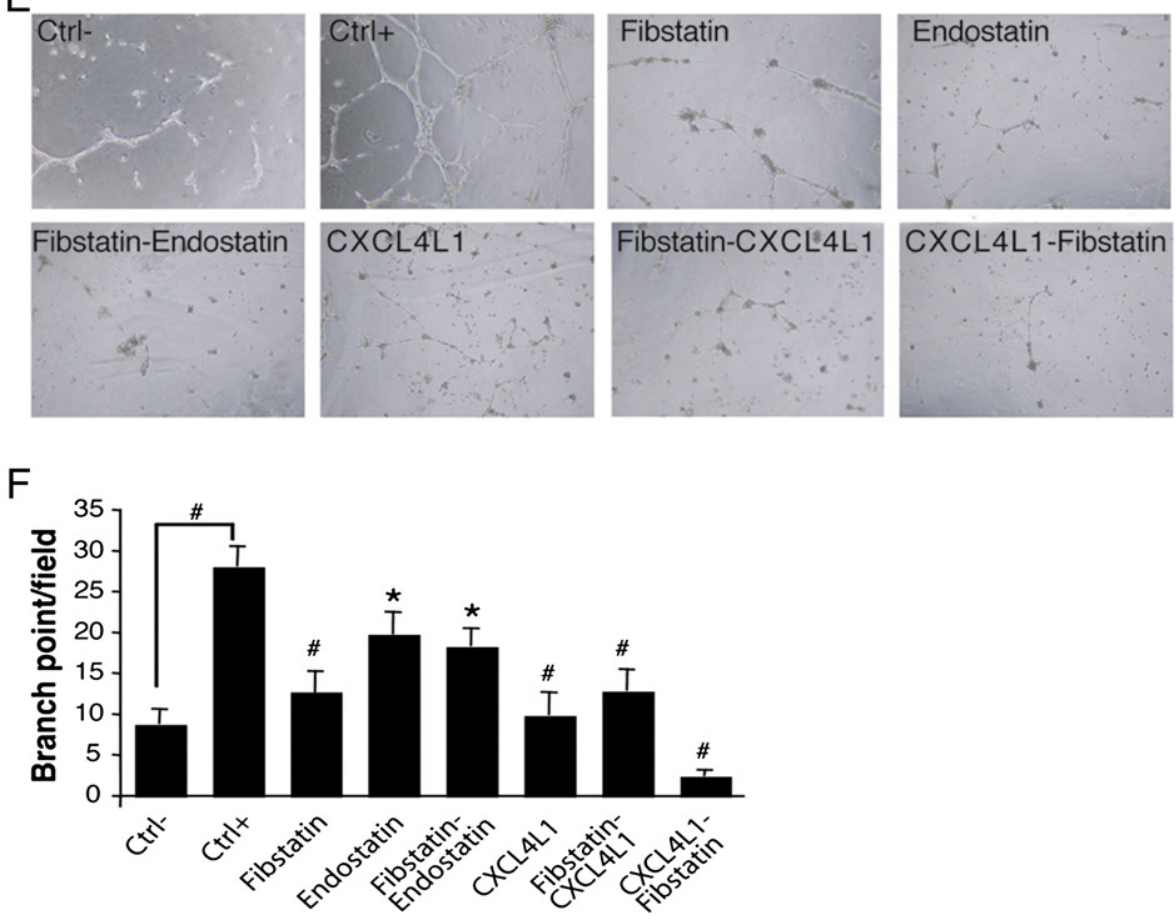

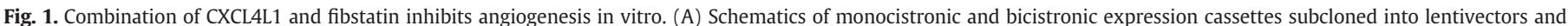

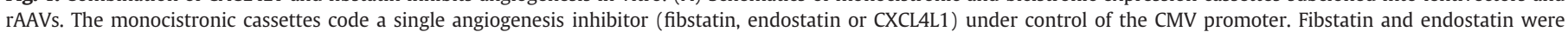

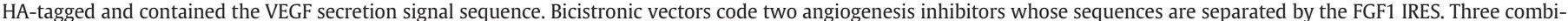

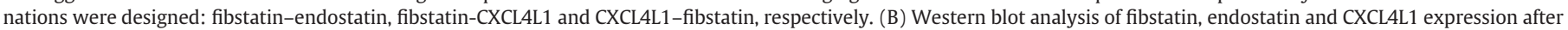

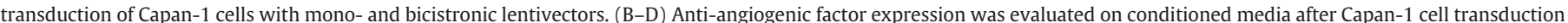

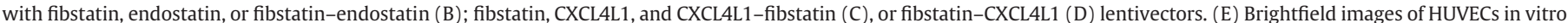

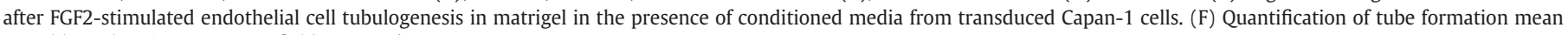
vessel branch points per $100 \times$ field \pm SEM. ${ }^{*} \mathrm{p}<0.01$, \#p $<0.001$.

CA) and $5 \mu \mathrm{m}$ tissue sections were immunostained with specific antibodies. Blood and lymphatic vessel numbers were quantified in 5-10 microscopic fields per cryosection by automated pixel density determination as the mean number of vessels \pm SEM for each treatment group. The mean number of mice with metastases in inguinal (Capan-1) lymph nodes was determined by immunostaining cryosections of nodes with $10 \mu \mathrm{g} / \mathrm{ml}$ anti pancytokeratin (TebuBio, Le Perray en Yvelines,
France). Detection was performed on 12 sections of lymph nodes, which means that one section every $80 \mu \mathrm{m}$ was analyzed.

Tumor studies

Capan-1 transduced cells $\left(2.10^{6}\right)$ were injected subcutaneously into NMRInu/nu mice $(n=10)$. Animals were sacrificed 2 weeks 
later and tumors were excised and embedded in OCT for histological analysis. For rAAV assay, $10^{6}$ tumor cells were injected 4 weeks after muscle transduction. Animals were sacrificed 28 days later and tumors and inguinal lymph nodes were excised. Experiments were performed three times.

\section{Statistical analysis}

All statistical analyses were performed with a two-tailed Student's t-test or ANOVA.

\section{Results}

Matrix proteolytic fragment fibstatin cooperates with Chemokine CXCL4L1, but not with endostatin, to inhibit in vitro angiogenesis

In order to test the effect anti-angiogenic factors combinations, lentivectors expressing either endostatin, fibstatin, and CXCL4L1 alone (monocistronic vectors), or combinations of these factors (bicistronic vectors) were generated (Fig. $1 \mathrm{~A}$ ).

Expression of fibstatin, endostatin, and CXCL4L1 was evaluated by Western blot in cell pellets and supernatants of Capan-1 cells transduced by the different lentivectors (Figs. 1B-D). Expression of fibstatin, endostatin or CXCL4L1 was easily detected from the monocistronic vectors (Figs. 1B-D). As regards the bicistronic vectors fibstatin-endostatin and fibstatin-CXCL4L1, they efficiently expressed the proteins from the first cistrons, whereas those expressed from the second cistrons were significantly less detectable (Figs. 1B-D). Therefore, two- and threetimes more proteins were loaded on the gel to detect the second cistron protein. We then produced fibstatin-CXCL4L1 and CXCL4-fibstatin lentivirus to evaluate the role of the cistron position in the biological effect (Figs. 1C,D). The control vectors were non-transduced (NT) cells and cells transduced by lentivector-GFP (Suppl. Fig. 2).

To evaluate the anti-angiogenic properties of the three molecules, alone or combined, we tested the ability of conditioned media from transduced Capan-1 to interfere with FGF2-induced endothelial cell tubulogenesis assay in Matrigel (Figs. 1E,F). When Capan-1 cells expressed one anti-angiogenic molecule, we observed a better inhibition of in vitro alignment with fibstatin or CXCL4L1 compared to endostatin (Fig. 1F). Fibstatin-endostatin and fibstatin-CXCL4L1 combinations had a similar effect as their monocistronic counterpart, probably due to the poor expression of the second cistron in these cells. In contrast the combination CXCL4L1-fibstatin, shown to co-express the two molecules efficiently (Figs. 1E,F), exhibited a strong inhibitory effect compared to CXCL4L1 or fibstatin alone. Surprisingly, when endothelial tubulogenesis was stimulated with VEGFA, no inhibition was observed with fibstatin demonstrating the FGF2-specificity (Suppl. Fig. 3). In this model, no synergistic effect was observed with fibstatin-endostatin vectors, whereas fibstatin-CXCL4L1 strongly inhibits tubulogenesis.

Fibstatin and CXCL4L1 exhibit an additive effect to inhibit endothelial cell migration

To dissect the anti-angiogenic effect of molecules in vitro, we evaluated the role of fibstatin, endostatin, and CXCL4L1, alone or combined, on FGF2-induced survival and migration of HUVECs in vitro. To analyze proliferation, we incubated HUVECs with conditioned media from Capan-1 transduced cells and anti-proliferative effects were assessed by the MTT assay and cell counting assay (Fig. 2A, Suppl. Fig. S4). As expected, endostatin, fibstatin and CXCL4L1 were able to suppress endothelial growth. We observed an additional effect on HUVEC survival of the combinations fibstatin-endostatin and CXCL4L1-fibstatin (Fig. 2A).

The effect of anti-angiogenic molecules on HUVEC migration was determined by the scratch wound assay (Figs. 2B,C). The three antiangiogenic agents inhibited HUVEC migration. However, no beneficial effect was observed with the combination fibstatin-endostatin. In contrast, association of CXCL4L1 and fibstatin generated more inhibition of HUVEC migration than the molecules alone (Figs. 2B,C). This was observed for both bicistronic vectors fibstatin-CXCL4L1 and CXCL4L1fibstatin.

These data demonstrated that fibstatin and CXCL4L1 was the most efficient combination to cooperate in vitro to block angiogenesis, and that this effect is due to inhibition of both proliferation and migration of endothelial cells (Fig. 2).

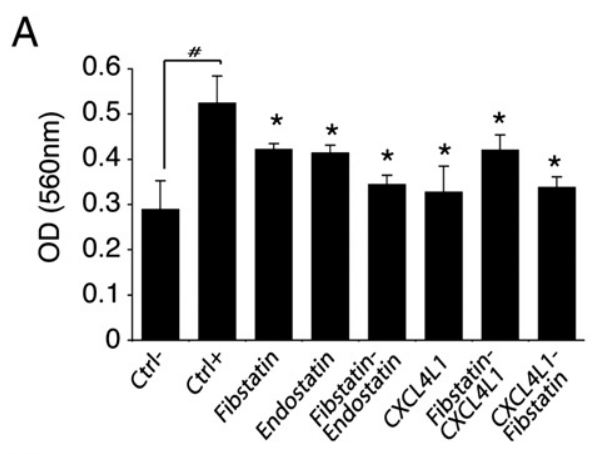

C

$\mathrm{B}$

Ctrl-

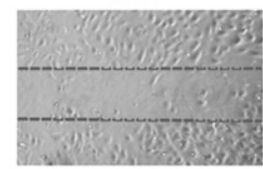

Fibstatin

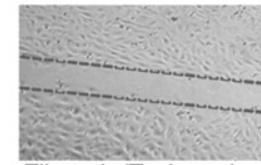

Fibstatin/Endostatin

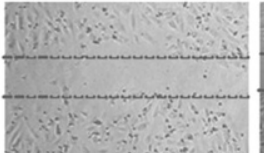

Fibstatin/CXCL4L1

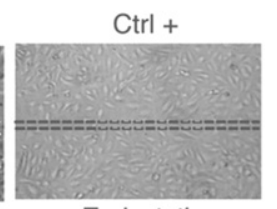

Endostatin

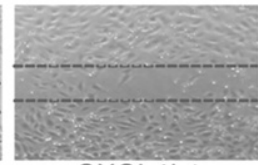

CXCL4L1

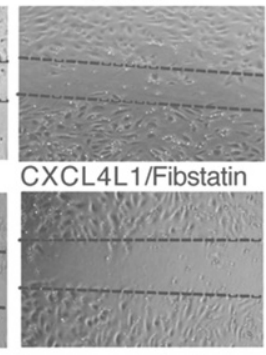

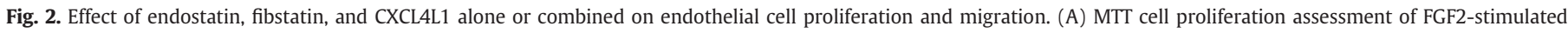

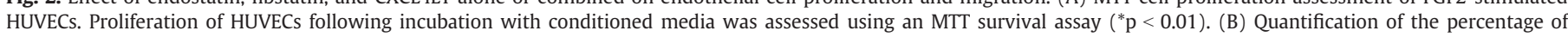

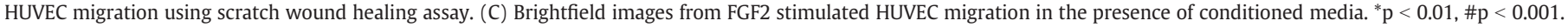


CXCL4L1 and fibstatin cooperate to inhibit tumoral angiogenesis but not tumor growth

To explore the additive effect of the anti-angiogenic molecules on tumor growth, we used local delivery of anti-angiogenic molecules in vivo. Capan-1 cells were transduced with the different lentivectors and subcutaneously injected in nude mice (Fig. 3A). After 7 and 14 days, tumor bearing mice were sacrificed and primary tumors were measured and removed for further analysis. We found that fibstatin, endostatin, and CXCL4L1 are able to inhibit tumor growth and tumor progression as well as the combinations fibstatin-endostatin and CXCL4L1-fibstatin (Fig. 3B). However, we did not observe a better anti-tumoral effect with the CXCL4L1-fibstatin combination compared with fibstatin-CXCL4L1.

Tumor sections were analyzed for angiogenesis by immunohistochemistry (Fig. 3C, Suppl. Fig. 5) and transgene expression in Capan-1 tumors was checked by RT-qPCR (Suppl. Fig. 6). We found that tumor-induced angiogenesis was substantially suppressed with all anti-angiogenic factors, alone or combined (Fig. 3C). The effect of the combination fibstatin-endostatin was similar to that of fibstatin or endostatin alone, whereas a significantly stronger anti-angiogenic effect was observed for the combination CXCL4L1-fibstatin, as observed for tubulogenesis in vitro (Figs. 1E-F).

These data showed that CXCL4L1 and fibstatin cooperate to block tumoral angiogenesis, but that this stronger angiogenesis inhibition has no beneficial effect on the blockade of tumor growth in the initial steps of tumor development (2 weeks).

Angiogenesis inhibition by CXCL4L1-fibstatin combination correlates with blockade of lymph node invasion

The absence of consequence of a stronger angiogenesis inhibition on tumor growth observed above for the CXCL4L1-fibstatin combination incited us to analyze tumor draining lymph node invasion. To target both primary tumor and metastatic loci, we performed rAAVs expressing mono- or bicistronic vector injections in the mice skeletal muscle of the leg. rAAVs expressing endostatin, fibstatin, CXCL4L1 or combinations of these factors were injected in the gastrocnemius and tibialis muscles four weeks before tumor xenograft (Fig. 4A). Tumoral angiogenesis was analyzed showing again that the CXCL4L1-fibstatin combination had the most efficient anti-angiogenic effect (Figs. 4B,C) compared to monocistronic vectors alone or fibstatin-endostatin combination. Tumor volume measurement showed, as for the local delivery, a strong antitumoral effect for all the rAAVs, but as observed using local delivery with lentivectors, no statistically significant additional inhibitory effect was observed for the CXCL4L1-fibstatin combination than for each anti-angiogenic factor alone (Fig. 4D).

To evaluate the effect of anti-angiogenic factor combinations on tumor lymph node invasion, inguinal draining lymph nodes were
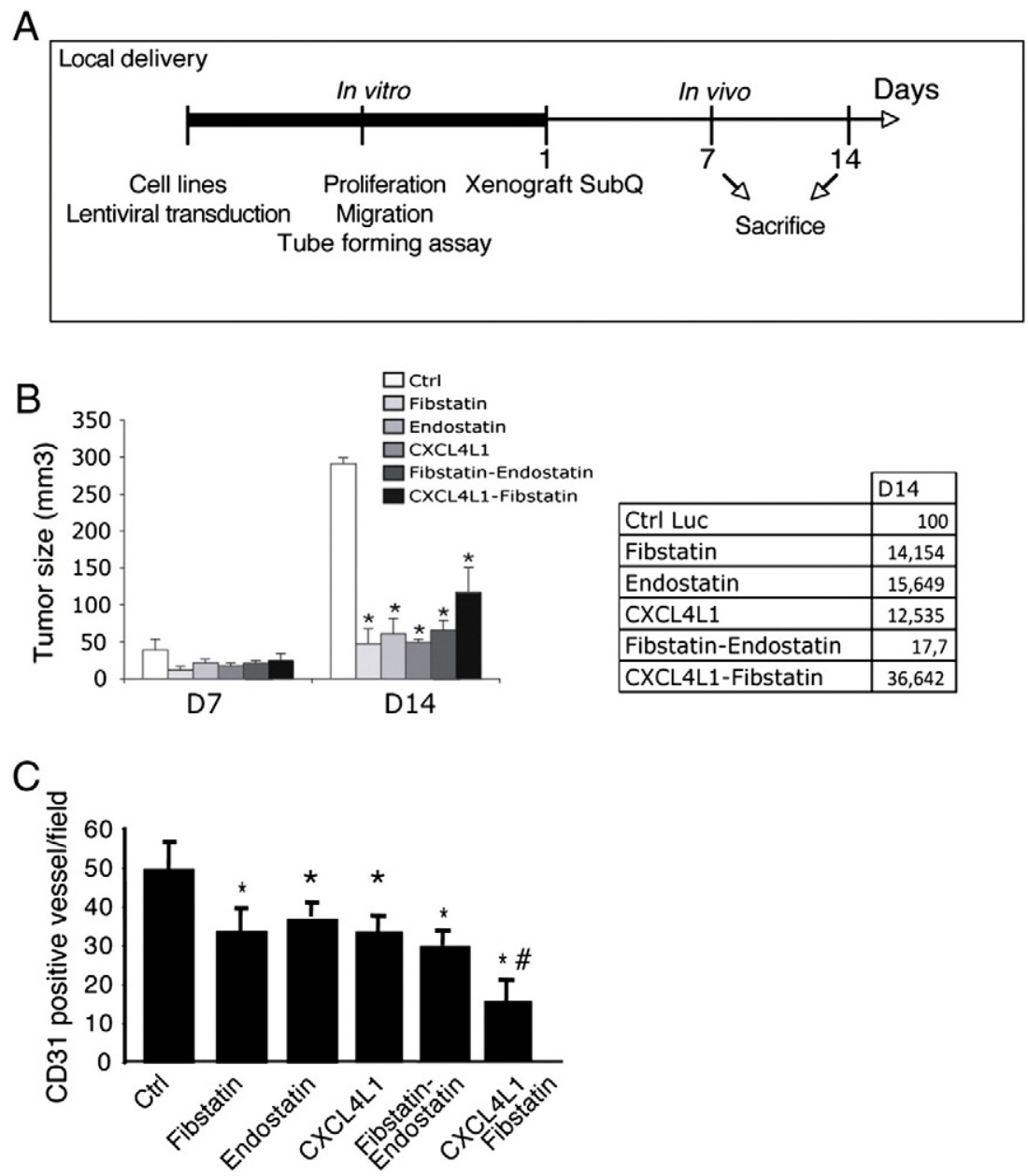

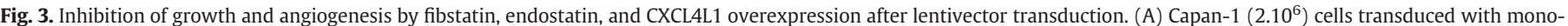

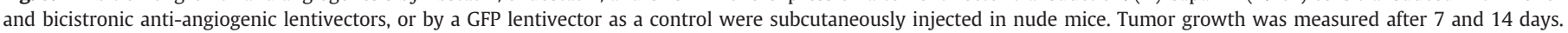

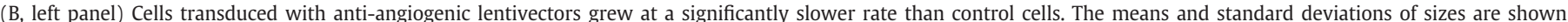

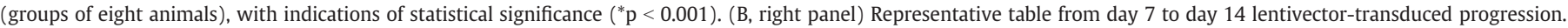

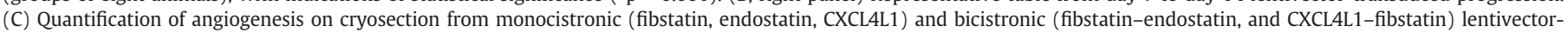
transduced pancreatic tumors (Capan-1). CD31-positive vessels \pm SEM per microscopic field quantified in replicate samples. ${ }^{*} \mathrm{p}<0.01$, \#p $<0.001$. 

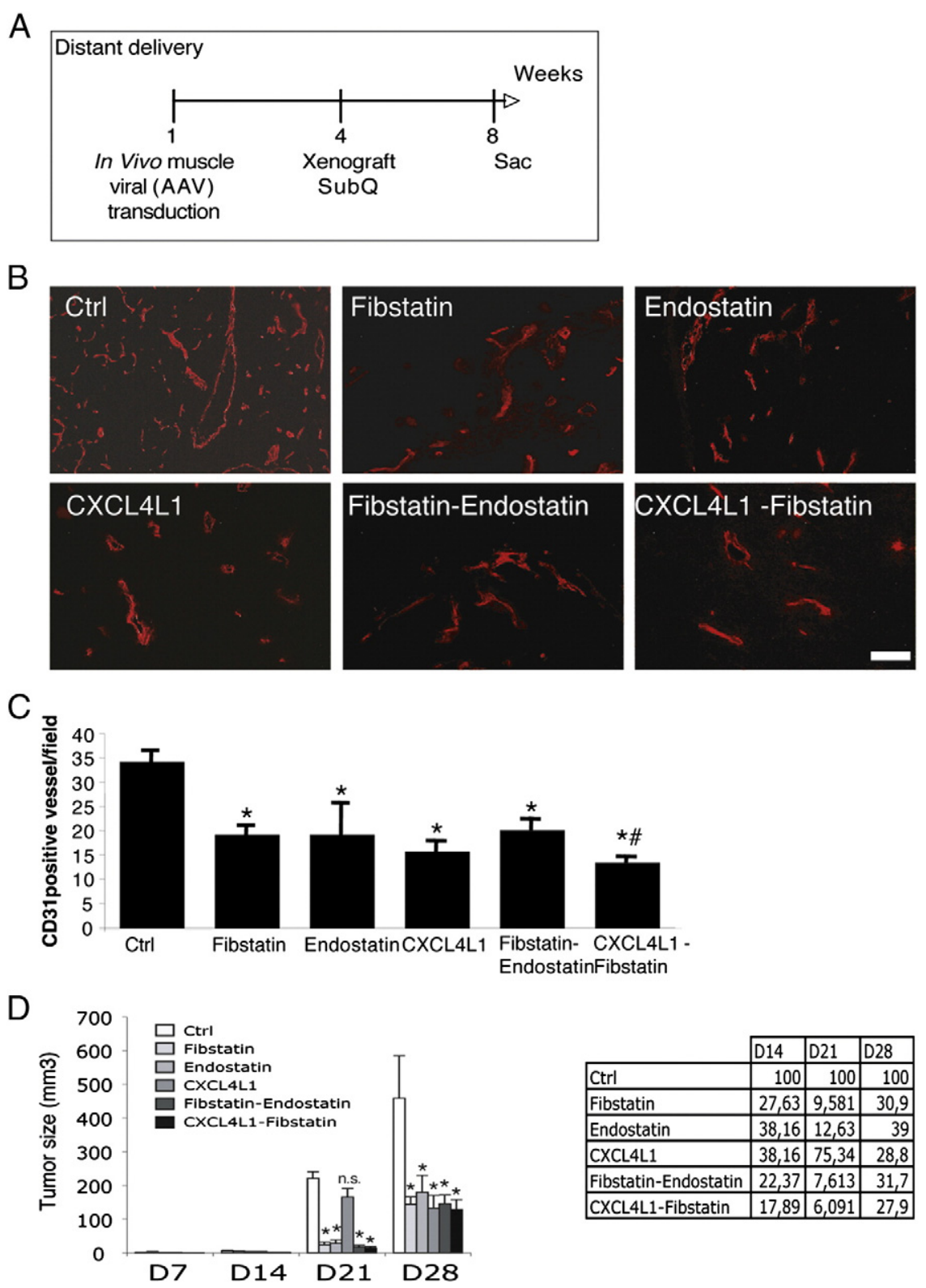

\begin{tabular}{|l|r|r|r|}
\cline { 2 - 4 } \multicolumn{1}{c|}{} & D14 & D21 & D28 \\
\hline Ctrl & 100 & 100 & 100 \\
\hline Fibstatin & 27,63 & 9,581 & 30,9 \\
\hline Endostatin & 38,16 & 12,63 & 39 \\
\hline CXCL4L1 & 38,16 & 75,34 & 28,8 \\
\hline Fibstatin-Endostatin & 22,37 & 7,613 & 31,7 \\
\hline CXCL4L1-Fibstatin & 17,89 & 6,091 & 27,9 \\
\hline
\end{tabular}

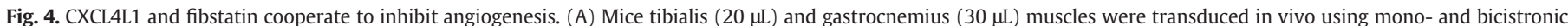

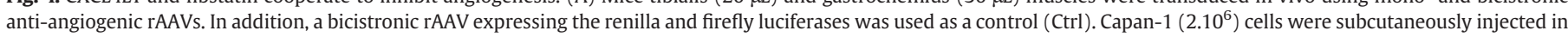

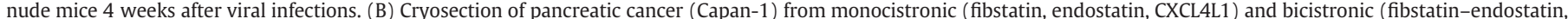

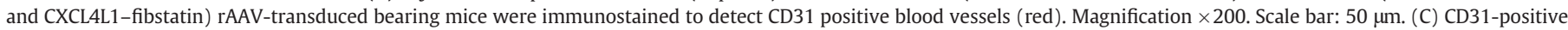

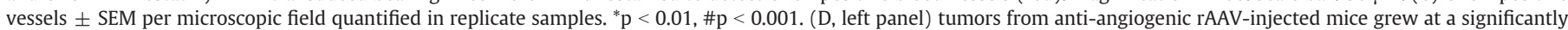

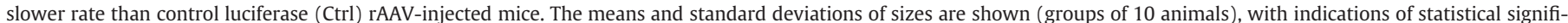
cance $\left({ }^{*} \mathrm{p}<0.001\right)$. (D, right panel) Representative table from day 14 to day 28 tumor progression.

analyzed four weeks after tumor injection and immunostained for cytokeratin positive tumor cells (Fig. 5A). We observed an inhibition of tumor cell invasion with all anti-angiogenic factors, alone or combined (Fig. 5A). However, we found a few clusters of cytokeratinpositive tumor cells in fibstatin-endostatin group, whereas no tumor cell was detected in CXCL4L1-fibstatin mice (Fig. 5B).

To determine a beneficial effect of the CXCL4L1-fibstatin combination compared to CXCL4L1 alone, we measured the level of expression of anti-angiogenic factors by Western blot on muscle protein extracts. This revealed a lower expression of anti-angiogenic factor expressed by the bicistronic AAVs, than by the monocistronic AAVs (Figs. 5C-F). In particular, CXCL4L1 and fibstatin expression were about 3 folds and 10 folds less expressed (Fig. 5F). These studies clearly indicated that CXCL4L1 associated with fibstatin is able to completely abolish tumor invasion at a lower dose than when administrated alone suggesting a synergistic effect of fibstatin and CXCL4L1, which is not observed for fibstatin and endostatin.

\section{Fibstatin and CXCL4L1 inhibit tumor lymphangiogenesis}

Lymphangiogenesis plays a crucial role in tumor dissemination to the lymph nodes. Recent studies have shown that endostatin inhibits both tumor angiogenesis and lymphangiogenesis, however nothing has been reported for fibstatin or CXCL4L1 (Brideau et al., 2007; Ou et al., 2011). To analyze the effect of these molecules, alone or combined, on tumor lymphangiogenesis in vivo, lentivector-transduced Capan- 1 cells were subcutaneously implanted into immunodeficient mice for two weeks. Tumors were removed and lymphangiogenesis 
A

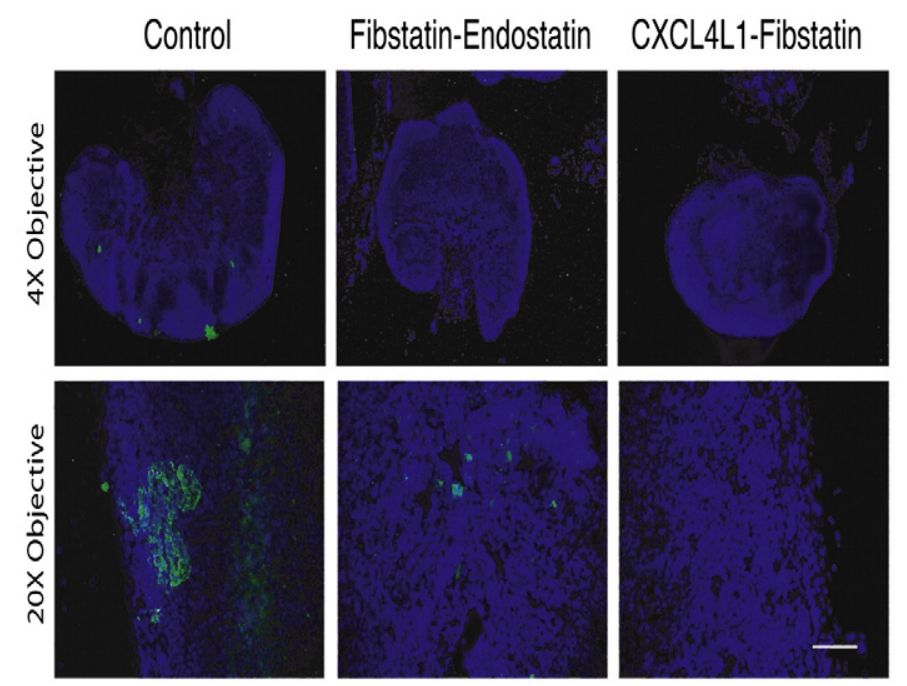

B
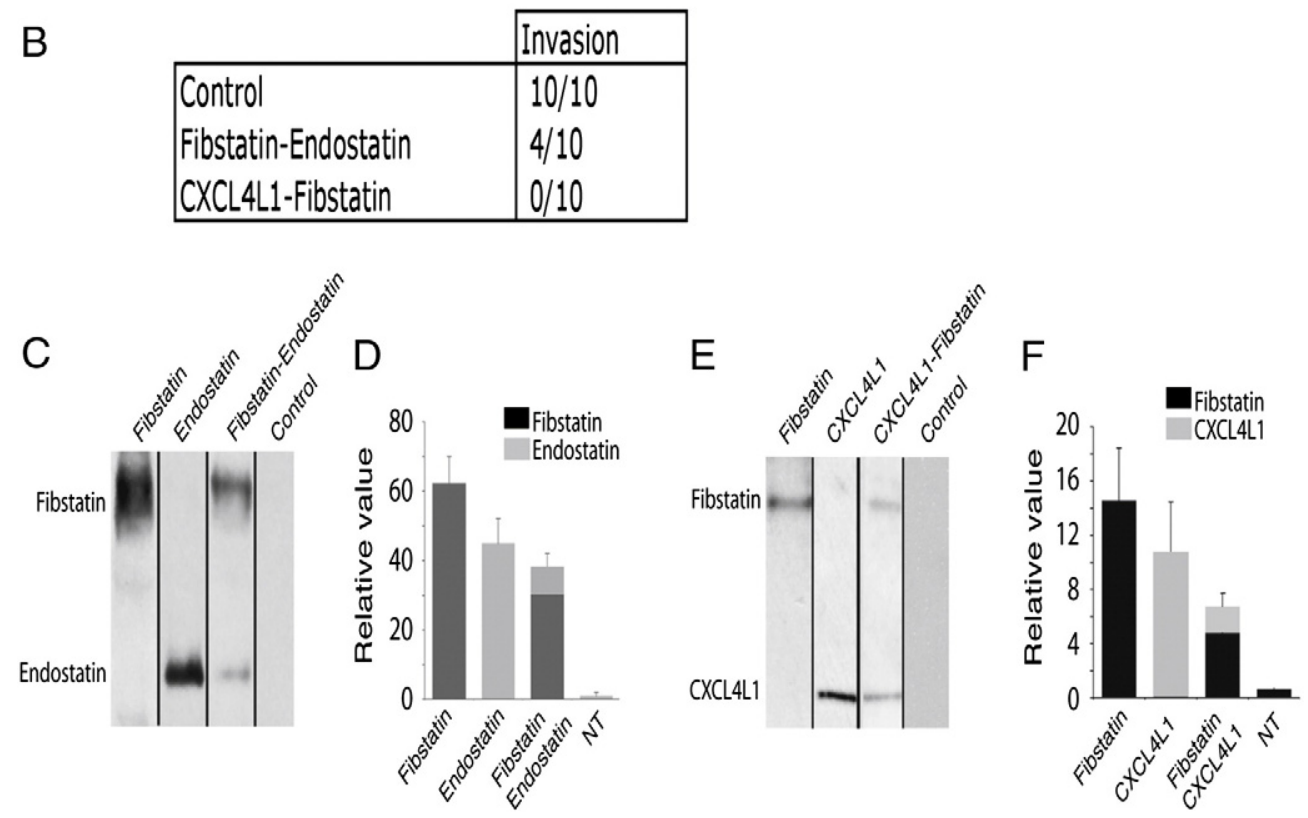

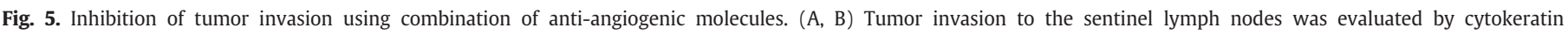

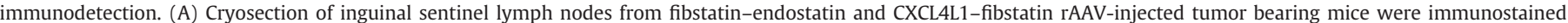

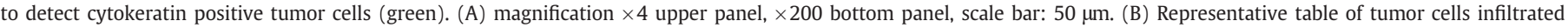

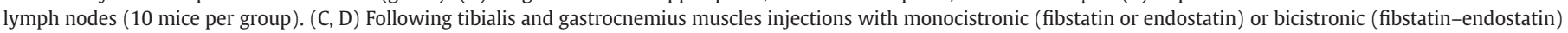

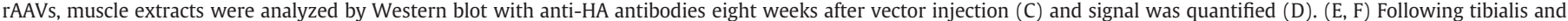

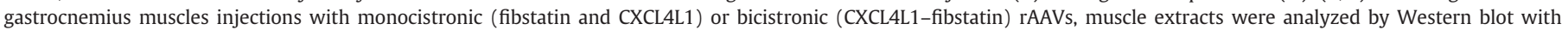
anti-HA and anti-CXCL4L1 antibodies eight weeks after vector injection (E) and signal was quantified (F).

was quantified after Lyve-1 immustaining (Fig. 6A, Suppl. Fig. S7) (Garmy-Susini et al., 2007). We found that tumor-induced lymphangiogenesis was substantially suppressed using monocistronic as well as bicistronic lentivectors (Fig. 6A). Interestingly, fibstatin and CXCL4L1 were more anti-lymphangiogenic than endostatin. In contrast to the data observed on angiogenesis, the combinations of molecules did not increase their effect on lymphangiogenesis.

When Capan-1 pancreatic carcinoma tumor cells were implanted subcutaneously in AAV-transduced mice, a strong inhibition of tumor lymphangiogenesis was observed with all the vectors, especially with the fibstatin. No better beneficial effect was detected using bicistronic versus monocistronic vectors (Figs. 6B-C). Nevertheless, as shown in Figs. 5C-F, we found a similar inhibitory effect of bicistronic vectors with lower amount of proteins, suggesting a cooperation of the molecules in the inhibition of lymphangiogenesis.
These data revealed fibstatin and CXCL4L1 as efficient antilymphangiogenic factors able to act upon local as well as distant delivery.

\section{Discussion}

This study reveals fibstatin and CXCL4L1 as new endogenous inhibitors of tumor lymphangiogenesis and metastasis. Furthermore, the combined administration of fibstatin and CXCL4L1 shows that they cooperate in the inhibition of tumoral angiogenesis. Interestingly, combined administration of the two molecules shows no beneficial effect on tumor growth, whereas it allows them to block tumor invasion in lymph node at lower doses.

Angiogenesis inhibitors for the treatment of cancer aim at depriving a tumor from developing the blood vessels and capillaries needed for 
A
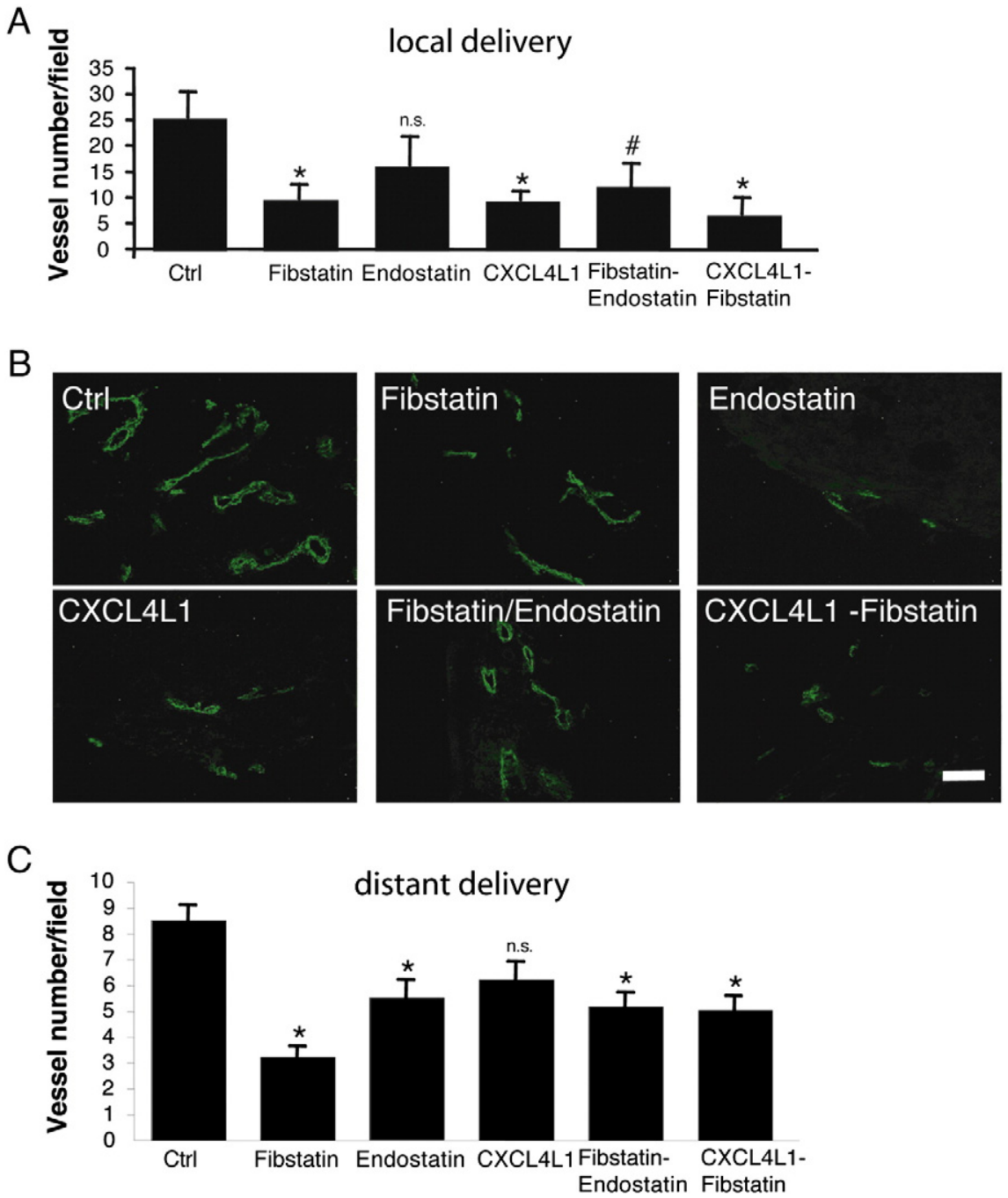

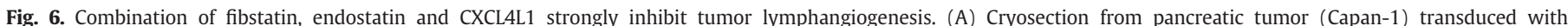

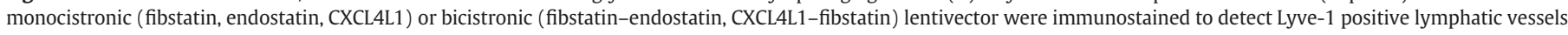

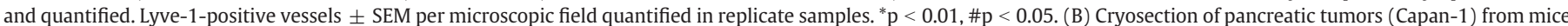

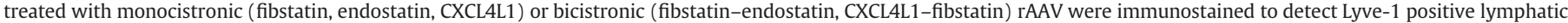
vessels (green). Magnification $\times 200$. Scale bar: $50 \mu \mathrm{m}$. (C) Lyve-1-positive vessels \pm SEM per microscopic field quantified in replicate samples. ${ }^{*} \mathrm{p}<0.01$.

further growth (Kerbel and Folkman, 2002). Nevertheless, progressionfree survival benefits from approved antiangiogenic drugs remain modest. Recent studies have shown that additionally to angiogenesis, lymphangiogenesis develops in primary tumors or in the peritumoral space and promotes lymphatic metastasis (Avraamides et al., 2008; Tammela and Alitalo, 2010). We show here that the anti-angiogenic factors used in our study exhibit both anti-angiogenic and antilymphangiogenic effects. However, we show that fibstatin and CXCL4L1 are stronger inhibitors of lymphangiogenesis than endostatin, which correlates with the inhibition of lymph node invasion using these molecules. This supports the crucial role of lymphangiogenesis in tumor invasion and underlines the importance of developing treatments against cancer that target lymphangiogenesis in addition to angiogenesis, to block metastasis dissemination.

The use of combined therapeutics has appeared for several years as a new concept to counteract the tumor cell ability to escape an antiangiogenic treatment (Byers and Heymach, 2007). The first combination to show its efficiency has been association of endostatin and angiostatin, either by plasmid transfection or viral vector transduction, or by using endostatin-angiostatin chimer (Campochiaro, 2011, 2012; Ponnazhagan et al., 2004). Such a combination appears as a promising therapeutic for ocular neovascularization diseases as well as for cancer therapeutics (Foy et al., 2011; Kim and Park, 2005). Another example of efficient combined treatment is the association of HER-2 and VEGF peptide mimics, which provides greater efficacy than individual treatments on breast tumor angiogenesis and growth (Foy et al., 2011). However the benefit of combining anti-angiogenic factors cannot be generalized to any molecule association. A previous study has reported the absence of benefit of combined delivery of anti-angiogenic agents $\mathrm{p} 53, \mathrm{GM}-\mathrm{CSF}$ and angiostatin, leading to the conclusion that they produce their anti-metastatic activity through a common anti-angiogenic pathway (Liu et al., 1999). Indeed our data also show that the cooperative effect is dependent on the nature of the combined molecules: we observe no cooperation between two extracellular matrix proteolysis fragments, endostatin and fibstatin, whereas the combination of fibstatin with the chemokine CXCL4L1 demonstrates its cooperative efficacy between inhibitors targeting at the same time tyrosine kinase and GPCR receptors (Liu et al., 1999). This strongly correlates with the hypothesis of Liu et al. (1999): anti-angiogenic factors cooperate if they act on different anti-angiogenic pathways.

Anti-cancer therapeutics by gene delivery is used in many clinical trials. According to the data of the Journal of Gene Medicine (http:// www.abedia.com/wiley), 1155 gene therapy clinical trials against cancer are ongoing in 2012, which represents $64.7 \%$ of all clinical 
trials in gene therapy. However, although the concept of combined therapeutic efficacy in cancer treatment is more and more accepted, very little data are available reporting the use of IRES-based vectors to co-express therapeutic molecules. A bicistronic rAAV coding angiostatin and endostatin with the encephalomyocarditis virus (EMCV) IRES has been shown to promote a long-term efficacy of transgene expression and antitumoral effect in a model of human ovary cell line (Ponnazhagan et al., 2004). However, this report does not mention the effect of the bicistronic vector on angiogenesis, lymphangiogenesis or metastasis dissemination. We have previously validated the principle of IRES-based vectors for gene therapy of limb ischemia in a mouse model, by showing that a plasmid containing the FGF1 IRES (very active in muscle) and co-expressing FGF2 and Cyr61, allows these molecules to act in synergy to promote therapeutic angiogenesis (Delluc-Clavieres et al., 2008; Rayssac et al., 2009). Here we validate the use of IRESbased vectors, i.e. rAAVs with the FGF1 IRES, for anti-(lymph)angiogenic and anti-metastatic therapeutics.

Pancreatic adenocarcinoma is characterized by local invasion of adjacent structures and early metastasis to the lymph nodes and the liver (Rupp and Linehan, 2009). Because of difficulties in early diagnosis and effective treatment, pancreatic cancer is often lethal, and particularly requires the development of new therapeutics able to target metastases (Gordis and Gold, 1984). Although little information is available about the efficiency of anti-angiogenic therapy in pancreas adenocarcinoma, it has been clearly shown that induction of angiogenesis and lymphangiogenesis facilitates the progression of pancreatic cancer (Cui et al., 2011; Mulder et al., 2010). Here we observe a strong inhibition of Capan-1 tumor angiogenesis and lymphangiogenesis with an optimal effect of the combination CXCL4L1-fibstatin. More importantly, we demonstrate that the anti-(lymph)angiogenic rAAVs clearly inhibit tumor metastasis to the lymph nodes with the synergistic effect of CXCL4L1-fibstatin on both macro- and micro-metastases.

\section{Conclusion}

In the light of our study, CXCL4L1 and fibstatin thus appear as new attractive candidates to be used in combination for the treatment of cancer and the IRES-based vector gene therapy represents a promising approach in the clinical setting to suppress the spread of tumors through the lymphatic system. Furthermore we demonstrate that intramuscular delivery of therapeutic molecules using rAAVs is definitively efficient to target both primary tumor and metastasis and provides a new therapeutic approach in highly metastatic cancers such as pancreatic cancer.

Supplementary data to this article can be found online at http:// dx.doi.org/10.1016/j.mvr.2013.05.005.

\section{Acknowledgments}

We thank Y. Barreira from the platform Anexplo Genotoul (Inserm US006, Toulouse, France) for outstanding technical assistance. We thank L. Buscail (Inserm U1037 vector facility, Toulouse France). All animal studies were performed with approval by the INSERM Institutional Animal Care and Use Committee. These studies have been made thanks to the use of I2MC Cytometry Shared Resources (Toulouse, France).

This work was supported by the Foundation pour la Recherche Medicale (FRM), the Ligue régionale contre le Cancer, the Association Francaise contre les Myopathies (AFM), the Association pour la Recherche contre le Cancer (ARC), the Conseil Régional MidiPyrénées, and the Foundation Avenir. A.R. had a fellowship from ARC and N.A. had a fellowship from AFM.

\section{References}

Avraamides, C.J., Garmy-Susini, B., et al., 2008. Integrins in angiogenesis and lymphangiogenesis. Nat. Rev. Cancer 8 (8), 604-617.

Bossard, C., Van den Berghe, L., et al., 2004. Antiangiogenic properties of fibstatin, an extracellular FGF-2-binding polypeptide. Cancer Res. 64 (20), 7507-7512.

Brideau, G., Makinen, M.J., et al., 2007. Endostatin overexpression inhibits lymphangiogenesis and lymph node metastasis in mice. Cancer Res. 67 (24), 11528-11535.

Byers, L.A., Heymach, J.V., 2007. Dual targeting of the vascular endothelial growth factor and epidermal growth factor receptor pathways: rationale and clinical applications for non-small-cell lung cancer. Clin. Lung Cancer 8 (Suppl. 2), S79-S85.

Campochiaro, P.A., 2011. Gene transfer for neovascular age-related macular degeneration. Hum. Gene Ther. 22 (5), 523-529.

Campochiaro, P.A., 2012. Gene transfer for ocular neovascularization and macular edema. Gene Ther. 19 (2), 121-126. http://dx.doi.org/10.1038/gt.2011.164 (Epub 2011 Nov 10) (Feb).

Cui, K., Zhao, W., et al., 2011. The CXCR4-CXCL12 pathway facilitates the progression of pancreatic cancer via induction of angiogenesis and lymphangiogenesis. J. Surg. Res. 171 (1), 143-150.

Delluc-Clavieres, A., Le Bec, C., et al., 2008. Efficient gene transfer in skeletal muscle with AAV-derived bicistronic vector using the FGF-1 IRES. Gene Ther. 15 (15), 1090-1098.

Dubrac, A., Quemener, C., et al., 2010. Functional divergence between 2 chemokines is conferred by single amino acid change. Blood 116 (22), 4703-4711.

Foy, K.C., Liu, Z., et al., 2011. Combination treatment with HER-2 and VEGF peptide mimics induces potent anti-tumor and anti-angiogenic responses in vitro and in vivo. J. Biol. Chem. 286 (15), 13626-13637.

Garmy-Susini, B., Makale, M., et al., 2007. Methods to study lymphatic vessel integrins. Methods Enzymol. 426, 415-438.

Garmy-Susini, B., Avraamides, C.J., et al., 2010. Integrin alpha4beta1 signaling is required for lymphangiogenesis and tumor metastasis. Cancer Res. 70 (8), 3042-3051.

Gordis, L., Gold, E.B., 1984. Epidemiology of pancreatic cancer. World J. Surg. 8 (6), 808-821.

Kerbel, R., Folkman, J., 2002. Clinical translation of angiogenesis inhibitors. Nat. Rev. Cancer 2 (10), 727-739.

Kim, K.S., Park, Y.S., 2005. Antitumor effects of angiostatin K1-3 and endostatin genes coadministered by the hydrodynamics-based transfection method. Oncol. Res. 15 (7-8), 343-350.

Liu, Y., Thor, A., et al., 1999. Systemic gene delivery expands the repertoire of effective antiangiogenic agents. J. Biol. Chem. 274 (19), 13338-13344.

Mulder, K., Koski, S., et al., 2010. Antiangiogenic agents in advanced gastrointestinal malignancies: past, present and a novel future. Oncotarget 1 (7), 515-529.

Mumprecht, V., Detmar, M., 2009. Lymphangiogenesis and cancer metastasis. J. Cell. Mol. Med. 13 (8A), 1405-1416.

Nagy, J.A., Vasile, E., et al., 2002. VEGF-A induces angiogenesis, arteriogenesis, lymphangiogenesis, and vascular malformations. Cold Spring Harb. Symp. Quant. Biol. 67, 227-237.

Nierodzik, M.L., Karpatkin, S., 2006. Thrombin induces tumor growth, metastasis, and angiogenesis: evidence for a thrombin-regulated dormant tumor phenotype. Cancer Cell 10 (5), 355-362.

O'Reilly, M.S., Boehm, T., et al., 1997. Endostatin: an endogenous inhibitor of angiogenesis and tumor growth. Cell 88 (2), 277-285.

Ou, J., Li, J., et al., 2011. Endostatin suppresses colorectal tumor-induced lymphangiogenesis by inhibiting expression of fibronectin extra domain $\mathrm{A}$ and integrin alpha9. J. Cell. Biochem. 112 (8), 2106-2114.

Paez-Ribes, M., Allen, E., et al., 2009. Antiangiogenic therapy elicits malignant progression of tumors to increased local invasion and distant metastasis. Cancer Cell 15 (3), 220-231.

Ponnazhagan, S., Mahendra, G., et al., 2004. Adeno-associated virus 2-mediated antiangiogenic cancer gene therapy: long-term efficacy of a vector encoding angiostatin and endostatin over vectors encoding a single factor. Cancer Res. 64 (5), 1781-1787.

Rayssac, A., Neveu, C., et al., 2009. IRES-based vector coexpressing FGF2 and Cyr61 provides synergistic and safe therapeutics of lower limb ischemia. Mol. Ther. 17 (12), 2010-2019.

Renyi-Vamos, F., Tovari, J., et al., 2005. Lymphangiogenesis correlates with lymph node metastasis, prognosis, and angiogenic phenotype in human non-small cell lung cancer. Clin. Cancer Res. 11 (20), 7344-7353.

Rupp, C.C., Linehan, D.C., 2009. Extended lymphadenectomy in the surgery of pancreatic adenocarcinoma and its relation to quality improvement issues. J. Surg. Oncol. 99 (4), 207-214

Sirven, A., Ravet, E., et al., 2001. Enhanced transgene expression in cord blood CD34(+)-derived hematopoietic cells, including developing T cells and NOD/SCID mouse repopulating cells, following transduction with modified trip lentiviral vectors. Mol. Ther. 3 (4), 438-448.

Skobe, M., Hawighorst, T., et al., 2001. Induction of tumor lymphangiogenesis by VEGF-C promotes breast cancer metastasis. Nat. Med. 7 (2), 192-198.

Stacker, S.A., Caesar, C., et al., 2001. VEGF-D promotes the metastatic spread of tumor cells via the lymphatics. Nat. Med. 7 (2), 186-191.

Struyf, S., Burdick, M.D., et al., 2007. Platelet factor-4 variant chemokine CXCL4L1 inhibits melanoma and lung carcinoma growth and metastasis by preventing angiogenesis. Cancer Res. 67 (12), 5940-5948.

Tammela, T., Alitalo, K., 2010. Lymphangiogenesis: molecular mechanisms and future promise. Cell 140 (4), 460-476. 